\title{
Is the failure to lose weight among the obese partly because obesity is an addictive disease?
}

\begin{abstract}
Obesity is a worldwide health issue that shows no signs of abating despite concerted efforts in the past decade to understand the determinants of this growing problem, as well as efforts to prevent or reduce the problem. Associated with high rates of morbidity, and lower than anticipated life quality and years of life, as well as significant impacts in middle life on working ability, the costs to the individual and society are increasingly exorbitant, with no respite in sight. This brief was designed to examine the most recent data in PUBMED and Web of Science that discusses the possible role played by foods that can become addictive in the context of obesity, and to examine the evidence base linking food addiction and obesity. Results show that despite the absence of an extensive research base, and clear differences between addictive drugs and foods, there is increasing evidence from human and animal-based research that food addictions can occur in the same way individuals can become addicted to chemical substances, and that a linkage to obesity in some cases cannot be overlooked.
\end{abstract}

Volume 4 Issue 3 - 2016

\author{
Ray Marks ${ }^{1,2}$ \\ 'Department of Health and Behavior Studies, Columbia \\ University, USA \\ ${ }^{2}$ Department of Health/Physical Education \& Gerontological \\ Studies/services, The City University of New York, USA
}

\begin{abstract}
Correspondence: Ray Marks, Department of Health and Behavior Studies, Columbia University, Teachers College, Box I I4, 525W 120th Street, New York, NY 10027, Tel I 126783445 , Fax 12126788259, Email rm226@columbia.edu
\end{abstract}

Received: December 20, 2015 | Published: February 19, 2016

Keywords: childhood obesity, food addiction, prevention

\section{Overview}

Obesity is a worldwide health issue that shows no signs of abating despite concerted efforts in the past decade to understand the determinants of this growing problem, as well as efforts to prevent or reduce the problem. Associated with high rates of morbidity, and lower than anticipated life quality and years of life, ${ }^{1}$ as well as significant impacts in middle life on working ability, ${ }^{2}$ the costs to the individual and society are increasingly exorbitant, with no respite in sight. So why do individuals become obese and why do the obese often fail to lose weight and maintain this, and why are rates of this problem increasing across the globe-within such a limited time frame? Does some form of addiction contribute to the onset, manifestation and inability to successfully change compulsive eating patterns without relapse in today's society where processed foods are engineered to enhance the desire for fat and sugar? If an addiction is a behavior repeated in face of contradictory evidence-in this case behaviors involving the overconsumption of specific food, ${ }^{3}$ the chronic use of certain foods and lack of engagement in physical activity - does this tend to result in weight gain, rather than weight loss or maintenance, followed by high levels of body dissatisfaction, poor self-concept, and reduced emotional wellbeing, plus other health, family, social, and quality of life problems as in the case of substance dependence? Similarly is the fact that the changes needed to reverse this situation are not always forthcoming or sustained when adopted ${ }^{4}$ due to similar neurological changes in certain brain pathways or structures. While addiction has a genetic basis, is it possible that the rise in obesity that prevails globally, is not due solely to changes in the gene pool, or lack of physical activity, but because some foods are engineered to provide comfort, or stimuli that are deemed satisfying to the brain, and that potentially tend to rewire the brain as with chemical substances, thus making them highly sought after, as proposed by Mogul et al. ${ }^{5}$

According to the American Society of Addiction Medicine, an addiction refers to a continual pursuit of a behavior that is increasingly harmful. ${ }^{6}$ In addition, accompanying alterations in the brain's reward system, motivation, and related pathways, can conceivably impact how an individual thinks, feels, and makes judgments. This situation can presumably affect their ability to engage in healthful activities, and to make careful decisions. According to Gearhardt et al., ${ }^{7}$ previous research has consequently supported the need for further examination of the apparent association between features of addiction to psychoactive substances and excess food intake.

While not as obvious as mainstream chemicals, certain foods that have addictive properties and that are highly marketed and part of the social norm may increase the struggle to lose weight, even if one or more of these food addictions results in a variety of irreversible health challenges, and social problems. Alternately, Topcu et al., ${ }^{8}$ found obese children may experience psychiatric disorders to a greater degree than normal weight children. According to this group, the heightened anxiety and depression of the obese children-could be due to their need to seek more comfort laden foods, but could also suggest a negative feedback cycle of obesity, heightened depression, and anxiety, fewer social interactions, and more challenges with energy balance along with emotion driven eating. Pretlow et al., ${ }^{9}$ found many respondents, ages 8 to 21, exhibited DSM-IV substance dependence (addiction) criteria when describing their relationship with highly pleasurable foods, suggesting obesity in children and adolescents may stem from possible addiction to highly pleasurable foods in youth. More recently, Schulte et al., ${ }^{10}$ concluded that highly processed foods may share characteristics with drugs that lead to abuse, especially if these are taken in frequently, and in high doses, such as fats and sugars as outlined by Lindberg et al., ${ }^{11}$ Moreover, due to the addition of fat or refined carbohydrates and the rapid rate the refined carbohydrates enter the system, certain foods may be implicated in addictive-like eating patterns.

Although somewhat refuted by Harb et al., ${ }^{12}$ who argued in favor of distinctive neurological process that differentiate food addiction from substance addiction-as a stronger obesity determinant than addiction, according to Burrows \& Meule ${ }^{3}$ the concept of a food addiction is not unlike an addiction to chemicals such as cocaine and other substances, and may result in a similar response to the consumption of certain high calorie, highly palatable energy dense, low nutrient content foods. 
Burrows \& Meule $^{3}$ found 4 studies dating back to 2009 that examined food addiction. In the most recent study reviewed, $38 \%$ of a sample of obese adolescent inpatients at a weight loss clinic could be said to have a food addiction. Pedram et al., ${ }^{13}$ found that those cases $5,4 \%$ of cases who were obese showed an increasing trend towards a food addiction with higher body mass among 652 adults. Further, those food addicts consumed more calories from protein and fat compared with controls, and clinical counts of food addiction correlated positively with body composition measures when the entire sample was observed.

\section{Objective}

This brief is designed to examine the most recent data that discusses the possible role played by foods that can become addictive, and can contribute to weight gain due to the frequency of ingestion, the type of foods that are possibly addictive, and the sedentary culture that limits the amount of calories expended after eating. The technological culture also anecdotally fosters snacking and exposure to food advertisements and evidence was sought to support future research to tease out whether more focused efforts on food policy, and awareness of this and food marketing, are desirable if these are implicated in the onset of addictive eating behaviors.

\section{Methods}

To achieve the present objectives, we focused largely on current research published in 2015 on this topic that could be identified in PUBMED and Web of Science using the key terms 'food addiction and obesity'. Previous work has been forthcoming up to 2013, and is well documented in Burrows \& Meule, ${ }^{3}$ so the goal was simply to examine if there is increasing evidence to support future work to identify the role played by food addiction in this multilayered problem of obesity, and if so, is the source of this addiction potentially amenable to intervention or treatment. All study types were deemed eligible, and those selected were chosen after screening if the topic directly addressed the theme of interest in this report. Excluded were articles related to the Food Addiction Scale, brain neurophysiology, binge eating disorder, stigma and addiction, and specific diseases and food addiction. The flow chart pertinent to the search is shown in Figure 1. All data were extracted and summarized in narrative form or tabulated to display the findings of the study. No synthesis was conducted due to the paucity of data in this area, the highly diverse study questions, samples, research designs, measures applied and topics covered, but several themes were covered, including food addiction and obesity, causes of food addiction, solutions for treating obesity in light of food addiction research findings (Figure 1).

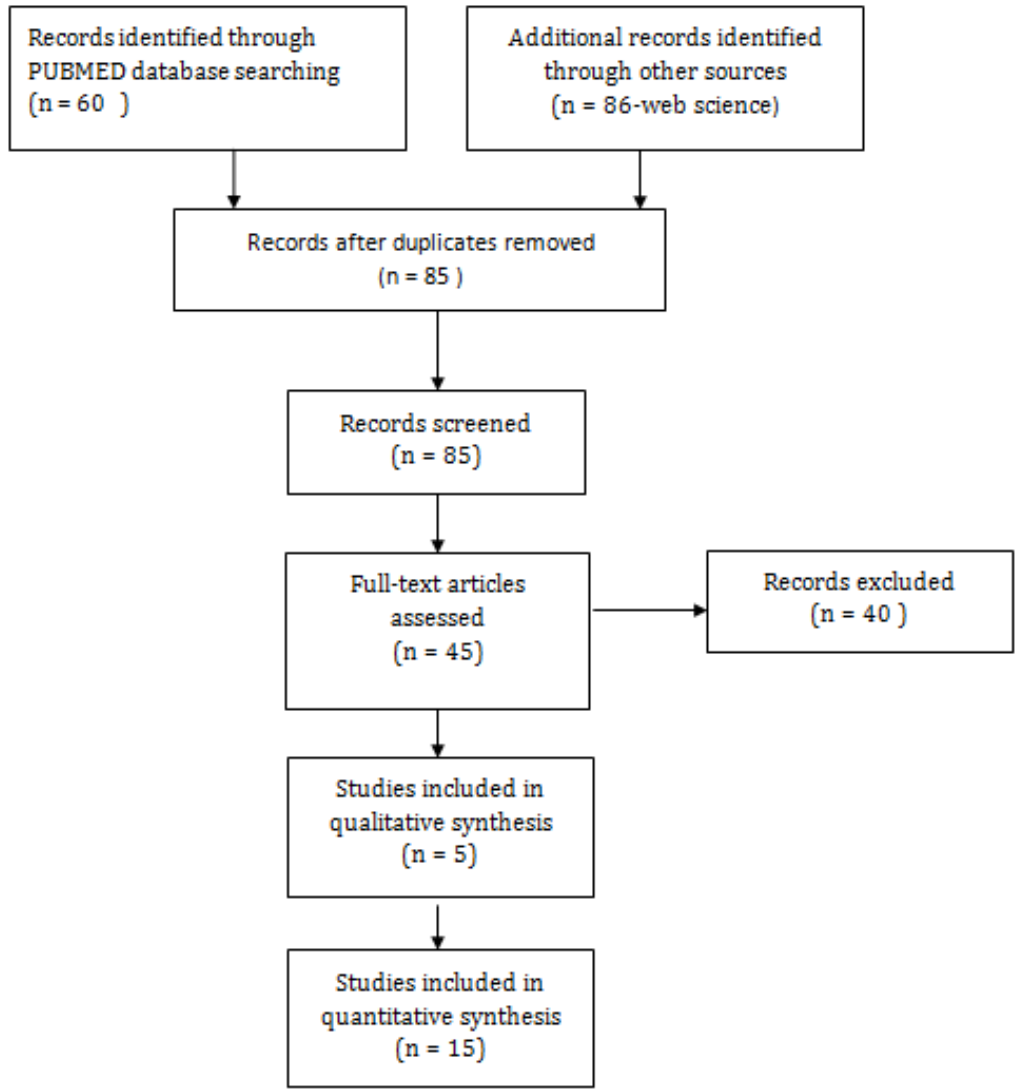

Figure I PRISMA 2009 flow diagram key words food addiction and obesity restricted to 2015.

\section{Results}

\section{Food addiction and obesity}

Although very current few articles of those listed on the data base were clearly focused on the topic of food addiction and obesity specifically, among the papers retrieved, which house references to the related literature, in particular, Meule ${ }^{14}$ show addictions to eating, as well as addictions to sugar and fat, and accompanying anxiety and depression, are potentially linked to overeating and some cases of obesity as discussed by Dimitrijevic et al. ${ }^{1}$ According to their review of some recent research findings, and as supported by results shown in Table 1, the aforementioned authors show how similarities 
can be made between individuals addicted to substances, and those addicted to food. In terms of treating the relevance of food addiction to obesity, Dimitrijevic et al., ${ }^{1}$ further argue that not only does excess food ingestion produce a greater risk of weight gain, but this may be hard to combat if an addicted individual cannot readily withdraw from food use as in drug withdrawal situations. Indeed, food addiction was shown to be a significant and problematic issue for children suffering from excess weight according to an earlier study by Merlo et al. ${ }^{22}$
As such, being able to identify the presence of food addiction among those who are overweight, may indicate some forms of obesity are potentially treatable, ${ }^{22}$ regardless of any genetic disposition towards obesity, and that most individuals with some degree of food addiction can be expected to manage the disease and reduce the instances of relapse with, support, stress management (if needed) and welldesigned behavioral modification.

Table I Studies on food addiction and obesity in the current PUBMED 2015 data base

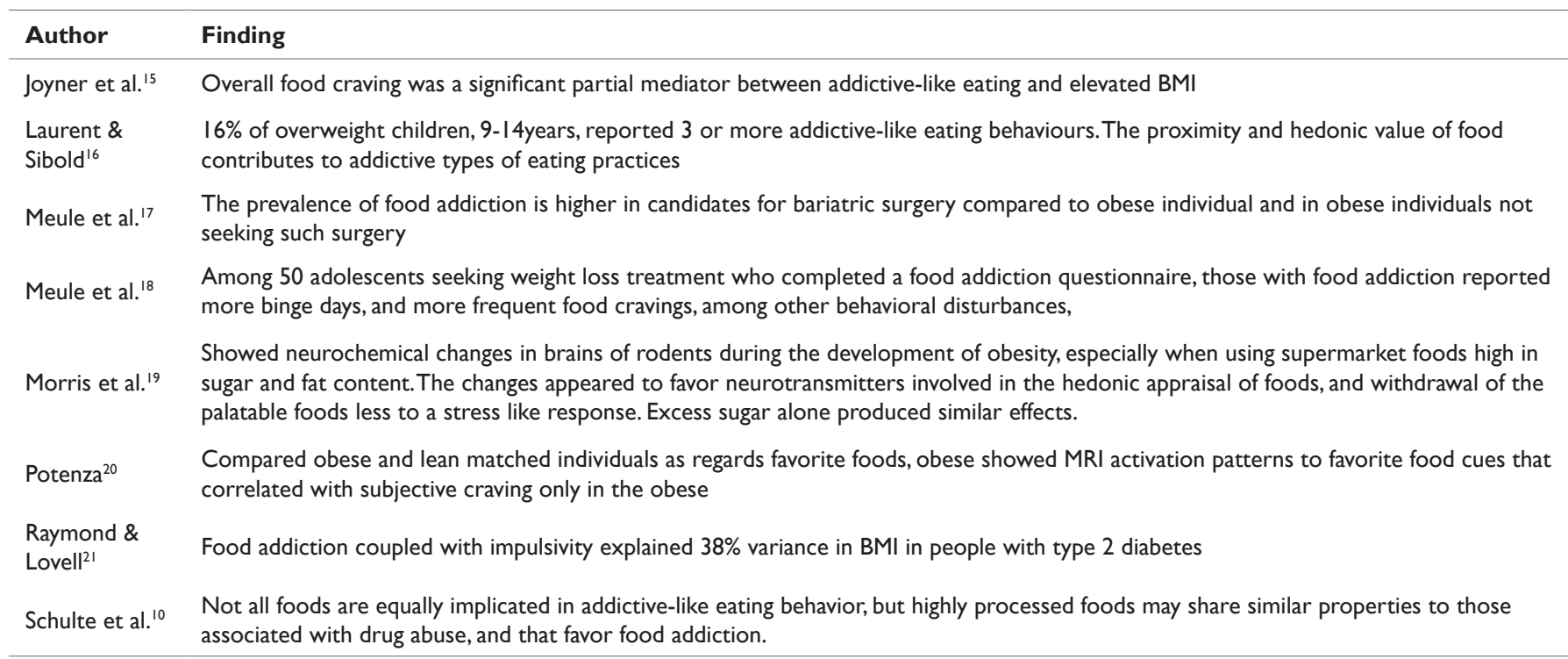

However, as Dimitijovic et al., ${ }^{1}$ reported, not only is the desire to stop food consumption often unsuccessful, and time consuming, but that once people are choosing hyperpalatable foods in excess, their desire for this grows, and they are found to readily reduce other activities in order to consume and purchase these foods. Moreover, there is not only continued use, but continued use in spite of observable health and personal problems associated with obesity and exposure to a nutrient poor processed food diet. In this respect, children in particular, may be highly vulnerable, especially those who have been conditioned early on to acquire a taste for fat and sugar, and/or those with reduced executive cognitive performance and brain alterations. ${ }^{23}$ This group found child overweight and obesity associated with reduced executive cognitive performance and MRI based brain alterations.

SwamaNantha report ${ }^{24}$ "food addiction" could lead to high levels of sugar consumption and excess sugar related morbidities after reviewing 94 reports on the topic of addiction. Robinson et al., ${ }^{25}$ who examined the role of wanting versus liking in motivating addictive behaviors, stated that excess 'wanting' and 'liking' for food, especially highly palatable foods may foster overeating, one of the primary causes of obesity. In addition food stimuli may attract high attention in those with higher body mass indices. They reported on one of their recent studies that showed food cues were excessively attractive only to rats fed on junk food. Those rats that over consumed the junk food and gained weight in large amounts initially displayed greater attraction for liking and wanting food related cues-prior to junk food exposure and seemed more willing to work for their presentation. Overtime there seemed to be a blunting in the liking response and a decoupling of liking and wanting. The cues for food have been shown to activate brain motivational regions and may play a role in weight maintenance according to these authors.

\section{Causes of the problem}

The problem of any addiction is not always strictly a genetic or biological one, but can often be attributed to environmental, cultural, and personal factors, as well as stress, and social circumstances, plus a reward system of the limbic brain area that drives the motivation to consume more palatable and energy-dense foods. ${ }^{26}$ In the case of food addictions, marketing of addictive foods, their low cost, and their availability all make the problem a highly explosive one. The presentation and packaging of fast food and other products that contain sugar, fat, and salt, and bombardment of food-associated cues that trigger intense motivation to seek food ${ }^{25}$ may encourage overeating, especially in low socioeconomic areas, and if the ingredients are refined and modified to increase their sensory properties and to activate the brain's reward system. ${ }^{25}$

Unsurprisingly, their ubiquity and palatability may encourage substance dependence according to Garber \& Lustig $^{27}$ who reviewed both the characteristics of fast food consumers and the presentation and packaging of fast foods. In terms of encouraging substance dependence this group found most fast food meals were accompanied by a soda, thus increasing the sugar content 10 -fold, an amount found to lead to sugar addiction, tolerance and withdrawal in animal models ${ }^{27}$ and food advertisements providing environmental cues that could favor food addiction were increasingly common. TV exposure through marketing to children, and the manufacture of hyperpalatable energy dense foods that do not produce satiation in small portions ${ }^{1,7}$ are additional possible overlapping determinants. 
According to Gearhardt et al., ${ }^{7}$ the same or similar brain sites activated by drugs, are activated by exposure to certain high caloric foods and sweet drinks in anticipation of eating, and neuroimaging studies provide evidence that obese individuals have altered characteristics of brain reward sites and exhibit tolerance attributes. ${ }^{27}$ As outlined by Zhang et al. ${ }^{28}$ there are some shared neural and hormonal pathways between foods eaten for pleasure and substances used to increase pleasure that may explain why some individuals continue to eat despite increasing negative consequences.

\section{Preventing obesity attributable to over eating}

As for the treatment for addiction, therapies ranging from inpatient treatment to outpatient treatment and support groups may be helpful depending on the problem manifested. An ecological approach that addresses policies that permit foods to be chemically engineered to produce addictions, and that are known to be unhealthy have not been successful, but need to be continued. Helping parents to become savvy consumers, and to be aware that food manufacturers are producing obesogenic foods that foster addiction ${ }^{29}$ is of great import, as is modeling healthful eating practices from the earliest point in time. Reducing the costs of healthful foods, and increasing their availability is clearly desirable as well.

Pretlow et al., ${ }^{9}$ suggest incorporating substance dependence methods may improve the success rate in combating the childhood obesity epidemic. However, as outlined by Shriner \& Gold, ${ }^{4}$ simplistic solutions must be ruled out if in fact the criteria for severe substance use disorders where six or more symptoms are indicative of a problem, are in any way applicable to those facing obesity issues.

a. The substance is often taken in larger amounts or over a longer period than was intended.

b. There is a persistent desire or unsuccessful efforts to cut down or control the use.

c. A great deal of time is spent in activities needed to obtain/use the substance.

d. A strong desire or urge to use the substance exists.

e. Recurrent use results in a failure to fulfill major roles at work, school, or home.

f. Continued use prevails despite persistent or recurrent social or interpersonal problems caused/increased by the substance.

g. Important social, occupation, or recreational activities are given up or reduced because of substance use.

h. Recurrent use in occurs despite the fact that usage is hazardous. ${ }^{30,31}$

Tolerance, as defined by either of the following may certainly occur as discussed by Dimirtijovic et al., ${ }^{1}$ even though this has not been studied in depth. That is, there may be:

I. A need for markedly increased amounts of the substance to achieve intoxication or desired effect-or amounts that increase the energy balance equation negatively.

II. A markedly diminished effect with continued use of the same amount of the substance.

III. Withdrawal, as manifested by either of the difficulty of the affected individual to maintain an eating plan other than the current one unless the impact on the brain reward system is factored into the equation

IV. The substance (or a closely related substance) is taken to relieve or avoid withdrawal symptoms or has some type of unique motivational value in areas of the brain involved in behavioral control, and there is less activation of that part of the brain that is involved in inhibitory actions. ${ }^{1}$

According to preliminary work in animal models, abstinence symptoms may arise in cases where tolerance to hyperpalatable foods has been established, and rats seemed to crave substances with similar effects, such as amphetamines. Zhang et al., ${ }^{28}$ discuss the possibility of the development of new pharmacologic interventions if food addiction can be examined more closely and with the application of functional resonance imaging to tease out how selected foods can activate brain reward systems incrementally, rather than inhibitory systems, among other factors.

Lindberg et al., ${ }^{11}$ who showed fat and sweeteners had direct effects on body mass index and were also the mediators of increased caloric consumption, have indicated that efforts to curb obesity rates should focus on the palatability of certain foods, and employ addictive models as a framework. Joyner et al., ${ }^{15}$ recently showed that while cravings for sweets and other carbohydrates significantly mediated the relationship between addictive-like eating and binge eating episodes, cravings for fats significantly mediated the relationship between addictive-like eating and elevated body mass index, suggesting a targeted approach focused on fat may be helpful.

\section{Discussion}

Childhood obesity has become an enormous challenge across the globe. ${ }^{22,32}$ Impacting both physical and psychological wellbeing one mechanism of obesity development that has not been well explored is that due to excess caloric intake as a result of being addicted to foods with high fat and sugar content as discussed by Merlo et al. ${ }^{22}$ In addition, data from animal models does suggest that the food addiction hypothesis in relation to obesity is potentially relevant. ${ }^{33}$

Other evidence does suggest that in addition to a possible increased preference for sweetness, ${ }^{22}$ contributing to this association may be various forms of stress, that have been correlated with emotion-driven eating, of high calorie and low nutrient food intake ${ }^{34}$ and the specific manufacture of obesogenic foods that fosters addiction..$^{29}$ It also seems noteworthy that obesity is more evident among the poorer classes who eat more foods in general that could be termed hyperpalatable, but low in nutrient value..$^{22}$

Consequently, regardless of the lack of a substantive research base in this area, and confusion as to whether foods can cause addictions similar to chemical substances, it seems reasonable to suggest more work in this realm is highly desirable given that solutions are imperative to stem the global obesity epidemic. Moreover, given that obesity treatment is commonly less effective than desired, or ineffective $e^{4}$ and research does show there is a very low probability of an obese person attaining a normal body weight as described by Fildes et al. ${ }^{35}$ exploring all avenues to uncover obesity-related mediating or moderating variables is highly recommended.

In sum, despite clear differences between addictive drugs and foods,${ }^{30}$ there is increasing evidence from human and animal-based research that food addictions can occur in the same way individuals can become addicted to chemical substances, and that the resultant 
behaviors of the food addicted person are not unlike those persons addicted to chemical substances making it challenging at the same time to intervene simply by dietary restrictions. It is possible that once foods are engineered to provide rewards not associated with traditional foods, along with their heightened availability, and low cost, access and exposure to fast food advertisements ${ }^{27}$ combine to produce behaviors are associated with possible weight gain as discussed by Gearhardt et al. ${ }^{30}$ Fortuna ${ }^{36}$ concluded that several biological and psychological similarities between food addiction and drug dependence exist, including loss of control and craving, while Garber \& Lustig $^{27}$ concluded that once obese, many cases do indeed meet criteria for psychological dependence.

\section{Conclusion}

The global obesity epidemic is more readily explained if we do not discount the overt manufacture of foods that are high fat and/or sugar content and known to contribute over time to the onset of obesity, and their availability worldwide, even though the link between being addicted to hyperpalatable foods and obesity is not clear cut as this may take considerable time to become evident. It is possible too that as with an addiction, the affected individual may deny there is a problem, or even if they recognize the problem, they fail to seek help, so practitioners are less aware of this issue than they might be. In addition, practitioners may not spend any time exploring this avenue or even be aware of screening measures for detecting this possible addiction. Vulnerable individuals, especially those who rely on fast foods to serve their nutritional needs, may not be aware of the potential problem this may cause as regards weight gain, and may thus perpetuate these eating behaviors even in the face of negative outcomes if they inadvertently become caught in a cycle of reward and positive feedback from some of these foods. They may also not be ready to change, and may thus continue their current behaviors despite increasingly negative consequences. Some, who begin behavioral interventions and notice improvements may quit these prematurely or relapse, because very careful long-term insightful intervention that accounts for the addictive properties of fast foods and others is not forthcoming.

Shriner \& Gold ${ }^{4}$ have gone so far as to propose three models to explain how food addiction can impact the onset of obesity, and are trying to extend this to modeling of the non-linear relationship of obesity and food addiction, due to the potential importance of the ramifications of foods with addictive properties. Their goal is to highlight how certain foods can potentially interact with other factors to foster excess consumption of hyperpalatable foods in an effort to develop effective interventions to counter obesity, and to contribute to the future research agenda in this realm.

In this respect, and as suggested by Lee et al. ${ }^{37}$ more research on the neurobiology of food addiction and its relationship to adolescent obesity in low- and middle-income groups, most at risk for obesity, would be especially helpful in revealing whether food marketing and manufacturing is not only encouraging hyper-ingestion of high density foods as discussed by Shriner \& Gold, ${ }^{4}$ but whether this behavior is linked clinically to the risk of obesity. As well, program evaluations that employ biochemical measures, and examine the relevance of food craving to obesity as outlined by Potenza \& Grilo ${ }^{38}$ are strongly recommended. According to Schwarz et al., ${ }^{39}$ given the current support for some sort of a mediating or moderating relationship to obesity in selected cases, a psychodynamically oriented approach to treating compulsive overeating, as well as an integrated model, rather than a medical model of intervention alone may be helpful for reducing obesity in selected cases. In addition, given the similarities of food addiction to other addictive behaviors Dimitrijovic et al., ${ }^{1}$ have advocated for the application of traditional addictive treatment approaches in the context of treating obesity, while a staged or incremental food withdrawal approach may be helpful as outlined by Pretlow et al. ${ }^{31}$

For similar reasons Cattivelli et al., ${ }^{40}$ propose that acceptance and commitment therapy may be helpful, while Shriner \& Gold ${ }^{4}$ stress integrating metabolic, addictive, and human relationship factors into obesity management efforts. Hebebrandet al., ${ }^{25}$ have argued that similar to smoking where efforts to limit advertising and to increase the price of smoking-and restrict access were successful, interventions to prevent food addiction might be successful if they follow similar strategies, despite the low validity of any food addiction claim. That is, structural efforts may be helpful even if food addiction is not a direct obesity cause, or only one of many related factors. ${ }^{41}$ Having the food industry on board as a player and adopting a holistic rather than targeted approach is also advocated. As well, Rogers ${ }^{42}$ propose it might be possible to "formulate foods that increase satiation and that can enhance attractiveness of lower-calorie, less energy-dense foods that can appeal in a similar way to sugars and other food substances that can activate the brain's reward system" [p. 1213].

While not yet proven, bearing in mind food preferences and eating practices are clearly largely socially constructed behaviors, and food cues and consumption can activate neural circuits that appear related to drug addiction ${ }^{30}$-exposing young people to a carefully conceived food selection and regimen, limiting their exposure to marketing messages of foods engineered to increase palatability, and helping them to make carefully informed decisions starting in their formative years is strongly indicated. Reducing stressors, and treating signs of depression and anxiety, and sleep deprivation, as well as possible signs of food addiction among young people, family communication interventions to improve children's unhealthy eating behaviors, using cognitive behavioral therapy and motivational interviewing is suggested ${ }^{5}$ to also likely to be advantageous. Creating a culture of health, and making healthy choices the easy choice is strongly indicated as well.

To protect children and those vulnerable to obesity, clinicians and others working with young people should make consistent, comprehensive concerted efforts to discourage hedonic eating, ${ }^{43}$ especially given that eating can become an addiction under certain circumstances, as with other behaviors. ${ }^{44}$ Some consideration that the brain is either impaired to start off with, or undergoes structural changes must be considered in light of the finding that despite it becoming increasingly known that added sugars can have detrimental effects on health, people persist in consuming them in excess. ${ }^{45}$

\section{Acknowledgements}

None.

\section{Conflict of interest}

The author declares no conflict of interest.

\section{References}

1. Dimitrijević I, Popović N, Sabljak V, et al. Food addiction-diagnosis and treatment. PsychiatrDanub. 2015;27(1):101-106. 
2. Nevanperä N, Ala-Mursula L, Seitsamo J, et al. Lasting obesity predicts poor work ability at midlife: a 15-year follow-up of the northern Finland 1966 birth cohort study. J Occup Environ Med. 2015;57(12):1262-1268.

3. Burrows T, Meule A. 'Food addiction'. What happens in childhood? Appetite. 2015;89:298-300.

4. Shriner R, Gold M. Food addiction: an evolving nonlinear science. Nutrients. 2014;6(11):5370-5391.

5. Mogul A, Irby MB, Skelton JA. A systematic review of pediatric obesity and family communication through the lens of addiction literature. Child Obes. 2014;10(3):197-206.

6. Definition of Addiction. American Society of Addiction Medicine.

7. Gearhardt AN, Corbin WR, Brownell KD. Preliminary validation of the Yale Food Addiction Scale. Appetite. 2009;52(2):430-436.

8. Topçu S, Orhon FŞ, Tayfun M, et al. Anxiety, depression and self-esteem levels in obese children: a case-control study. J Pediatr Endocrinol Metab. 2016;29(3):357-361.

9. Pretlow RA. Addiction to highly pleasurable food as a cause of the childhood obesity epidemic: a qualitative Internet study. Eat Disord. 2011;19(4):295-307.

10. Schulte EM, Avena NM, Gearhardt AN. Which foods may be addictive? The roles of processing, fat content and glycemic load. PLoS One. 2015;10(2):e0117959.

11. Lindberg MA, Dementieva Y, Cavender J. Why Has the BMI gone up so drastically in the last 35years? J Addict Med. 2011;5(4):272-278.

12. Harb MR, Almeida OF. Pavlovian conditioning and cross-sensitization studies raise challenges to the hypothesis that overeating is an addictive behavior. Transl Psychiatry. 2014;4:e387.

13. Pedram P, Wadden D, Amini P, et al. Food addiction: its prevalence and significant association with obesity in the general population. PLoS One. 2013;8(9):e74832.

14. Meule A. Back by popular demand: a narrative review on the history of food addiction research. Yale J Biol Med. 2015;88(3):295-302.

15. Joyner MA, Gearhardt AN, White MA. Food craving as a mediator between addictive-like eating and problematic eating outcomes. Eat Behav. 2015;19:98-101.

16. Laurent JS, Sibold J. Addictive-like eating, body mass index and psychological correlates in a community sample of preadolescents. $J$ Pediatr Health Care. 2016;30(3):216-223.

17. Meule A, Heckel D, Jurowich CF, et sl. Correlates of food addiction in obese individuals seeking bariatric surgery. Clin Obes. 2014;4(4):228236 .

18. Meule A, Hermann T, Kübler A. Food addiction in overweight and obese adolescents seeking weight-loss treatment. Eur Eat Disord Rev. 2015;23(3):193-198

19. Morris MJ, Beilharz JE, Maniam J, et al. Why is obesity such a problem in the $21^{\text {st }}$ century? The intersection of palatable food, cues and reward pathways, stress, and cognition. Neurosci Biobehav Rev. 2015;58:36-45.

20. Potenza MN. Obesity, food and addiction: emerging neuroscience and clinical and public health implications. Neuropsychopharmacology. 2014;39(1):249-250.

21. Raymond KL, Lovell GP. Food addiction symptomology, impulsivity, mood and body mass index in people with type two diabetes. Appetite. 2015;95:383-389.

22. Merlo LJ, Klingman C, Malasanos TH, et al. Exploration of food addiction in pediatric patients: A preliminary investigation. $J$ Addict Med. 2009;3(1):26-32
23. Bauer CC, Moreno B, González-Santos L, et al. Child overweight and obesity are associated with reduced executive cognitive performance and brain alterations:a magnetic resonance imaging study in Mexican children. Pediatr Obes. 2015;10(3):196-204.

24. SwarnaNantha Y. Addiction to sugar and its link to health morbidity: a primer for newer primary care and public health initiatives in Malaysia. J Prim Care Community Health. 2014;5(4):263-270.

25. Robinson MJ, Fischer AM, Ahuja A, et al. Roles of "wanting" and "liking" in motivating behavior: gambling, food and drug addictions. Curr Top Behav Neurosci. 2016;27:105-36.

26. Hebebrand J. Obesity prevention: moving beyond the food addiction debate. J Neuroendocrinol. 2015;27(9):737-738.

27. Garber AK, Lustig RH. Is fast food addictive? Curr Drug Abuse Rev. 2011;4(3):146-162.

28. Zhang Y, von Deneen KM, Tian J, et al. Food addiction and neuroimaging. Curr Pharm Des. 2011;17(12):1149-1157.

29. Lifshitz F, Lifshitz JZ. Globesity:the root causes of the obesity epidemic in the USA and now worldwide. Pediatr Endocrinol Rev. 2014;12(1):1734

30. Gearhardt AN, Grilo CM, DiLeone RJ, et al. Can food be addictive? Public health and policy implications. Addiction. 2011;106(7):12081212.

31. Pretlow RA, Stock CM, Allison S, et al. Treatment of child/adolescent obesity using the addiction model: a smartphone app pilot study. Child Obes. 2015;11(3):248-259.

32. Sahoo K, Sahoo B, Choudhury AK, et al. Childhood obesity: causes and consequences. J Family Med Prim Care. 2015;4(2):187-192.

33. Hone-Blanchet A, Fecteau S. Overlap of food addiction and substance use disorders definitions: analysis of animal and human studies. Neuropharmacology. 2014;85:81-90.

34. Tate EB, Spruijt-Metz D, Pickering TA, et al. Two facets of stress and indirect effects on child diet through emotion-driven eating. Eat Behav. 2015;18:84-90.

35. Fildes A, Charlton J, Rudisill C, et al. Probability of an obese person attaining normal body weight: cohort study using electronic health records. Am J Public Health. 2015;105(9):e54-e59.

36. Fortuna JL. The obesity epidemic and food addiction: clinical similarities to drug dependence. J Psychoactive Drugs. 2012;44(1):56-63.

37. Lee A, Gibbs SE. Neurobiology of food addiction and adolescent obesity prevention in low- and middle-income countries. J Adolesc Health. 2013;52(Suppl 2):S39-S42.

38. Potenza MN, Grilo CM. How relevant is food craving to obesity and its treatment? Front Psychiatry. 2014;5:164.

39. Schwartz DC, Nickow MS, Arseneau R, et al. A substance called food long-term psychodynamic group treatment for compulsive overeating. Int J Group Psychother. 2015;65(3):386-409.

40. Cattivelli R, Pietrabissa G, Ceccarini M, et al. ACT on FOOD: opportunities of ACT to address food addiction. Front Psychol. 2015;6:396.

41. Gearhardt AN, Grilo CM, DiLeone RJ, et al. Can food be addictive? Public health and policy implications. Addiction. 2011;106(7):12081212.

42. Rogers PJ. Obesity-is food addiction to blame? Addiction 2011;106(7):1213-1214.

43. Ziauddeen $\mathrm{H}$, Alonso-Alonso M, Hill JO, et al. Obesity and the neurocognitive basis of food reward and the control of intake. Adv Nutr. $2015 ; 6(4): 474-486$. 
44. Hebebrand J, Albayrak Ö, Adan R, et al. "Eating addiction" rather than "food addiction", better captures addictive-like eating behavior. Neurosci Biobehav Rev. 2014;47:295-306.
45. Avena NM, Potenza MN, Gold MS. Why are we consuming so much sugar despite knowing too much can harm us? JAMA Intern Med. 2015;75(1):145-146. 